\title{
Clostridium Difficile Infection Complicated By Toxic Megacolon In Immunocompetent Patient
}

${ }^{1}$ Medicine and Pharmacy Research Center, "Dunărea de Jos" University of Galati, Faculty of Medicine

${ }^{2}$ University "Ovidius" of Constanta, Faculty of Medicine

${ }^{3}$ Academy of Romanian Scientists

\begin{abstract}
Toxic megacolon can be a form of severe clinical course of the infection with Clostridium difficile (ICD), lifethreatening, requiring a particular course of treatment. Infection with Clostridium difficile in the Galati Infectious Disease Hospital presents rising number of cases, namely 172 cases in 2014, 271 cases in 2015 and 301 cases in 2016 with clinical evolutions with different severity degrees, including toxic megacolon and death. Among 744 patients with ICD in our clinic, since 1st January 2014 to 31 December 2016. The frequency of toxic megacolon (TM) was $0,537 \%$, so: 3 toxic megacolon cases with favorable evolution with treatment with vancomycin and metronidazole and just one case whose evolution was aggravated under this therapy and evolved favorably under treatment with tigecycline. The work presents this last case of ICD occurred in a 69 years old, immunocompetent man with unknown concomitant chronic diseases which undergoes surgery for bilateral inguinal hernia and receives antibiotherapy with cephalosporin IIIrd generation during surgery and after 7 days develops medium degree ICD with score Atlas 3 and receives therapy with oral vancomycin. He presents clinical aggravation during this therapy with the occurrence of colon dilatation, ascites and right pleurisy at ultrasound and therapy associated with metronidazole is decided. Clinical aggravation continues in this combined therapy
\end{abstract}

Liliana Baroiu

Lunca Siretului str. no. 43 A, Galaţi

email : lilibaroiu@gmail.com

phone: +40723201241 with defining the clinical, colonoscopy and tomography criteria for TM and is decided surgical monitoring and replacing antibiotherapy with tigecycline. Evolution is favorable with tigecycline without surgical intervention.

Keywords: Clostridium difficile, toxic megacolon, tigecycline.

\section{Introduction}

Infection with Clostridium difficile is directly related to the destruction of the microbiome after antibiotherapy (especially fluoroquinolone, cephalosporin, clindamicyn). In 2011 Centers for Disease Control and Prevention declares ICD a matter of public health and the main side effect after treatment with medication in the hospitals from the United States [1]. This thing occurred after the registration in 2011 in the United States of 500.000 ICD cases with 29.000 deaths in the first 30 days after diagnosis and 83.000 cases which presented recurrence. Moreover, the cost of a patient with ICD was estimated in the United States at approximately 35.000 American dollars and the ICD cost for the entire American health system at 3 billion dollars [1].

Clostridium difficile is a Gram-positive bacillus, anaerobe, formed of spores, first identified in 1935 (Hall and O'Toole) in the flora of the healthy newborns' colon [1]. It was considered non-pathogenic until 1978 (Bartlett and colleagues) $[2,3]$, when it was identified as a pathogenic agent of pseudomembranous colitis to a patient pre-treated 
with antibiotics. Polymerase chain reaction (PCR) detection of tcdB (toxin B Clostridium difficile) was first reported by Gumerlock in 1993 [4].

The epidemiology of this disease has dramatically changed in the past years with important increases of incidence and severity of cases reported in the United States and Europe [1]. This modification was assigned to the occurrence of a Clostridium difficile (CD) hypervirulent strain associated with the epidemics from Canada, USA, Europe $[1,5,6,7]$ - (BI/ NAP1/027), rapidly spreading strain, with a mutation in a negative regulator of the toxin production which leads us to an increase in the toxin production. This strain also synthesizes an additional toxin, it has an increased ability to generate germs and is highly resistant in comparison to the historical strain [1] Other strains are also cited, such as the one identified in a Polish study, ribotype 046, resistant to rifampicin, present in patients with ICD and antituberculous treatment [8].

Several studies performed in Romania demonstrate that the main circulating strain is ribotype $027[9,10]$.

The clinical ICD list includes diarrhea (minimum 3 stools a day with reduced consistency 5-7 Bristol scale), ileus (constipation and vomits with enteral distensia) and toxic megacolon.

Toxic megacolon associated with ICD has an incidence of $0.4-3 \%[11,12]$ and a mortality rate of $38-80 \%[12,13]$.

There are available several studies on TM in South-East of Romania: the Constanta Infectious Disease Hospital reports 3 ICD cases with TM out of 408 ICD cases (1.36\%), treated between 2014-2016, determined with $\mathrm{CD}$ ribotype 027, among which one case evolved favorably with therapy associated with vancomycin-metronidazole and two cases with vancomycin-metronidazole-tigecycline [14].

The Galati Infectious Disease Hospital treated 744 ICD cases, among which 4 toxic megacolon $(0.537 \%)$ [15].

Toxic megacolon represents the total or partial dilatation of the colon, non-obstructive, over $6 \mathrm{~cm}$ in diameter, associated with systemic toxicity signs. Toxic megacolon was initially described in 1950 by Marshak and Lester [16], and Jalan, in 1967 [17], defines the three mandatory diagnosis criteria:
A - radiologic - transverse or ascending colon dilatation over $6 \mathrm{~cm}$ in diameter;

B - three criteria of the following - fever over 38.6 degrees Celsius, heart rate over 120/min, leukocytosis over $10500 / \mu 1$, anemia;

$\mathrm{C}$ - one criteria of the following - dehydration, confusion, electrolyte abnormality, hypotension.

Toxic megacolon was initially described as a complication of ulcerative colitis, then in various forms of colitis: inflammatory, ischemic, infectious, after $\mathrm{x}$-ray, unspecific colitis secondary to chemotherapy $[18,19]$, complication of the collagenous colitis $[20,21]$, colitis from the Behcet syndrome [22,23], Kaposi syndrome [24] and pseudomembranous colitis after therapy with methotrexate.

The potential germs involved in the TM etiology are: Salmonella spp., Shigella spp., Campylobacter spp., Yersinia spp., Clostridium difficile [11,12,25], Entamoeba histolytica, Cryptosporidium [26], Cytomegalovirus [27], Rotavirus [28], Aspergillus spp. [29].

Moreover, they have been mentioned to create the risk of TM: abrupt discontinuities in therapy with steroids, sulfasalazine, aminosalicylic acid 5, as well as medication with negative impact on the intestinal motility (anticholinergics, antidepressives, loperamide, opioids). [30].

Anatomopathologically, in TM from ICD, parietal inflammation seems to affect the muscles and determine neuronal injury, leading to motility and dilatation disorders [12].

\section{Case presentation}

We present the case of a Caucasian man, admitted in November 2014 in the Galati Infectious Diseases, 69 years old, living in urban environment, retired, no chronic affections, immunocompetent, undergone surgery for bilateral inguinal hernia.

The patient received antibiotherapy with a dose of cephalosporin IIIrd generation before surgery and 
tree days after surgery and discharge from hospital seven days before ICD onset. The disease occurred 5 days before being admitted in the Infectious Diseases Hospital.

At admission, the patient had 5-6 liquid stools/ day, anorexia, nausea, vomiting, dry tongue, apyretic, diffuse abdominal pain, tachycardia, normal blood pressure (BP), body mass index 23.2, leukocytosis 31.350/U1 (normal-4000-10000), neutrophils 82\% (normal-50-62\%)- 25.710/Ul (normal 2000-7500), no anemia, fibrinogen $709 \mathrm{mg} / \mathrm{dl}$ (normal 180-350), erythrocyte sedimentation rate- $36 \mathrm{~mm} / \mathrm{h}(\mathrm{n}-0-15)$, toxin A Clostridium difficile negative, toxin B-positive (Certest Biotec-immunochromatographic method), coproculture in aerobiosis and uroculture negative (table 1).

Vancomycin is administered $250 \mathrm{mg}$ every 6 hours, it is considered a medium severity case, ATLAS score 3 (age over 60, leukocytosis over 25000/UI).

After 7 days, clinical evolution is unfavorable and the patient presents: 6 liquid stools/day, subfebrility, 9790/U1 leucocytes, 72,9\% neutrophils, erythrocyte sedimentation rate (esr) $-19 \mathrm{~mm} / \mathrm{h}$, fibrinogen-376mg/dl, negative procalcitonin, C protein reactive positive, serum albumin-2,18g/ $\mathrm{dl}$, toxin $\mathrm{A}$ and $\mathrm{B}$ Clostridium difficile low positive (immunochromatography).

Abdominal ultrasound reveals colon with thickened diffuse walls, intraperitoneal fluid in small amount, right pleural collection in small amount.

Treatment is changed as follows: vancomycin $500 \mathrm{mg}$ every 6 hours, metronidazole $500 \mathrm{mg}$ every 8 hours and the case is considered severe (ATLAS score 4-age over 60 years, fever - 37.9 degrees Celsius, serum albumin $2.18 \mathrm{~g} / \mathrm{dl}$ ).

Clinical evolution remains unfavorable and in 9 days the patient presents: 8 liquid stools/day, marked asthenia, diffuse abdominal pain, leucocytes-6710/ UI, neutrophils - 47\%, ATLAS score 5- age over 60 years, fever -38.6 degrees Celsius, serum albumin $1.97 \mathrm{~g} / \mathrm{dl}$ Systemic Inflammatory Response Syndrome - SIRS - fever, tachycardia).

Colonoscopy reveals: rectum mucous and colon with multiple inflammatory areas, covered by yellow residue, possibly fibrin, with form of pseudostenosing at hepatic angle, suggestive for colitis with clostridium difficile.
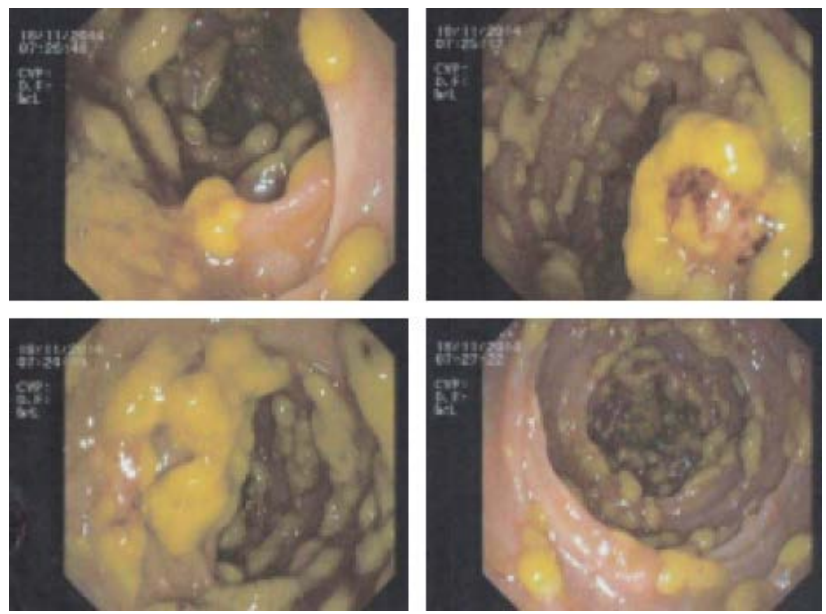

Figure 1 Colonoscopy image - Clostridium Difficile infection, inflammatory mucous with areas covered by yellow residue 1-4 mm

Abdominal-pelvic cat scan with contrast agent reveals: right pleural minimum liquid reaction, circumferential parietal thickness throughout the colon, also affecting the last ileal ansa, modifications of inflammatory type in the near peritoneal tissue. Free liquid in the peritoneal cavity under the liver, in the right parietal-colic space and in the small basin between anses. No pathological retroperitoneal adenopathies or other pathological modifications. Radiological diagnosis: important colon inflammatory modifications and ascites.

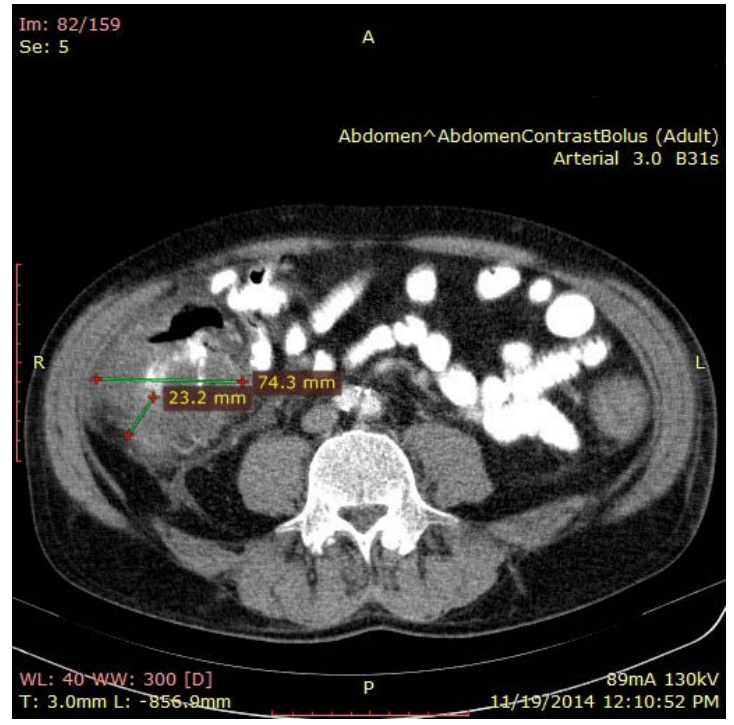

Figure 2. Ascending colon-wall thickness $23.2 \mathrm{~mm}$, transversal diameter $74.3 \mathrm{~mm}$. 


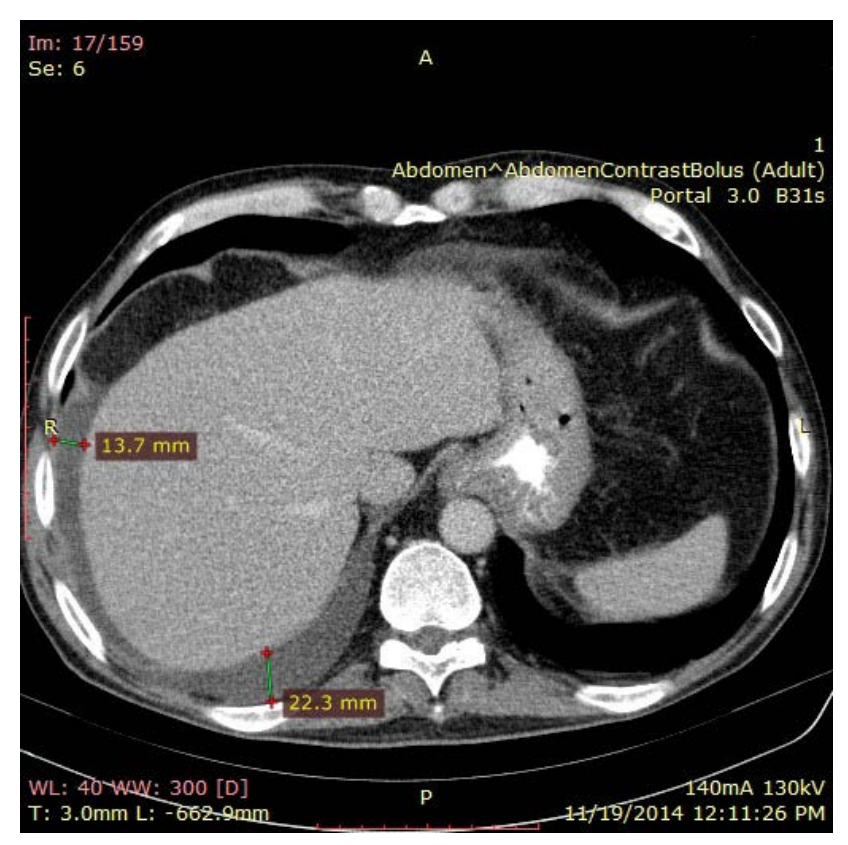

Figure 3. Pleural collection - liquid edge thickness 22.3 $\mathrm{mm}$, peritoneal collection, liquid edge thickness $13.7 \mathrm{~mm}$

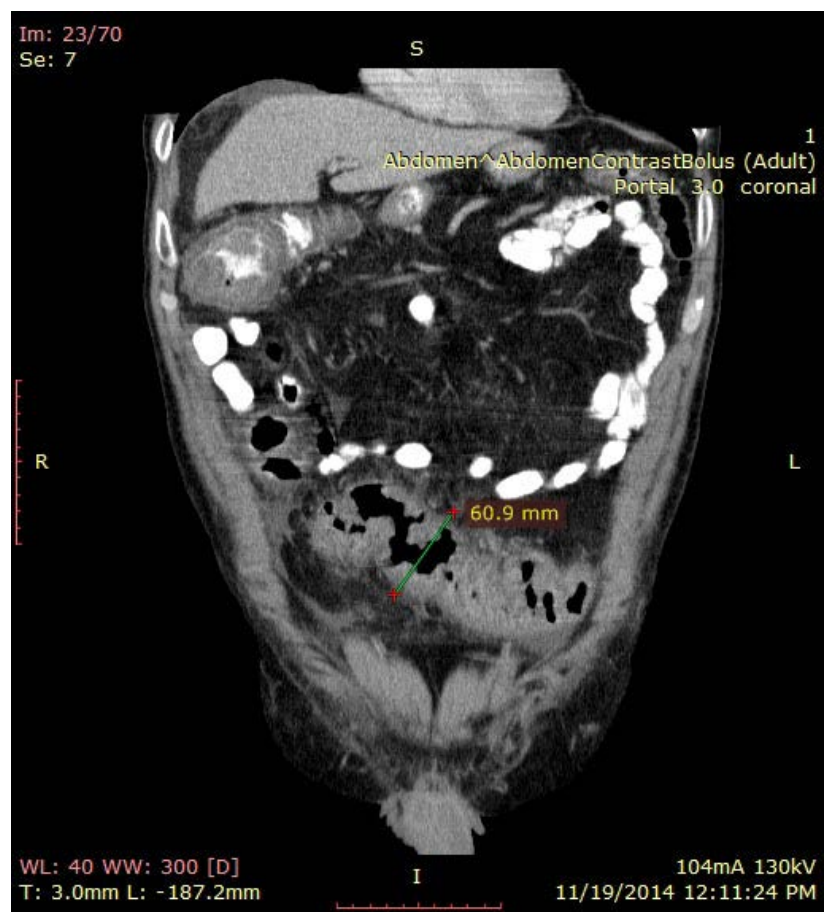

Figure 4. Sigmoid colon - diameter 60.9 mm(coronal section)

Table 1. Clinical-biological report

\begin{tabular}{|c|c|c|c|c|}
\hline & $5^{\text {th }}$ day of disease & $\begin{array}{l}12^{\text {th }} \text { day of } \\
\text { disease }\end{array}$ & $\begin{array}{l}21^{\text {st }} \text { day of } \\
\text { disease }\end{array}$ & $35^{\text {th }}$ day of disease \\
\hline \multicolumn{5}{|l|}{ Clinical raport } \\
\hline Number of stools & 5 liquid & 6 liquid & 8 liquid & 1 solid \\
\hline $\begin{array}{l}\text { Temperature (degrees } \\
\text { Celsius) }\end{array}$ & 36.4 & 37.9 & 38.6 & 36.5 \\
\hline $\mathrm{HR} * / \mathrm{min}$ & 130 & 93 & 90 & 77 \\
\hline $\mathrm{BP}^{* *}(\mathrm{~mm} \mathrm{Hg})$ & $120 / 80$ & $100 / 70$ & $90 / 60$ & $130 / 65$ \\
\hline \multicolumn{5}{|l|}{ Biological raport } \\
\hline Toxin A CD & negative & positive & & \\
\hline Toxin B CD & positive & positive & & \\
\hline $\begin{array}{l}\text { Number of leucocytes } \\
\text { (normal 4000-10.000/uL) }\end{array}$ & 31350 & 9790 & 6710 & 8111 \\
\hline $\begin{array}{l}\text { Hgb } \\
\text { (normal } 13-17 \mathrm{~g} / \mathrm{dl} \text { ) }\end{array}$ & 15.1 & 14.81 & 13.91 & 12.04 \\
\hline (normal $39-50 \%$ ) & 46.4 & 44.54 & 41.6 & 34.62 \\
\hline Serum albumine $(\mathrm{g} / \mathrm{dl})$ & & 2.18 & 1.97 & \\
\hline $\begin{array}{l}\text { Serum Na } \\
\text { (normal Na135-148 mmol/l) }\end{array}$ & 136 & 134 & 132 & 141 \\
\hline $\begin{array}{l}\text { Serum K } \\
\text { (normal K 3,5-5,3 mmol/1) }\end{array}$ & 3.9 & 4.2 & 3.4 & 4.4 \\
\hline$(\mathrm{Cl} 98-107 \mathrm{~mol} / \mathrm{l})$ & 101 & 105 & 105 & 110 \\
\hline
\end{tabular}

*HR: heart rate; **BP:blood pressure systolic/diastolic 
Previous treatment with antibiotics is cancelled and tigecycline is initiated - $100 \mathrm{mg}$ first dose, then 50 mg every 12 hours (ATLAS score 5 ).

In the $5^{\text {th }}$ day of treatment with tigecycline, the patient becomes apyretic with 2 semi-solid stools/day, leucocytes $6.980 / \mathrm{UI}$, neutrophils $28,8 \%$, esr- $4 \mathrm{~mm} / \mathrm{h}$, serum albumine- $1,97 \mathrm{~g} / \mathrm{dl}$.

After 11 days of treatment with tigecycline 1 solid stool/day, soft painless abdomen, leucocytes $-8110 /$ UI, neutrophils $66,9 \%$.

The patient is discharged after 14 days of treatment with tigecycline, after 3 days of normal stool.

\section{Discussion}

This ICD case, immunocompetent men, no comorbidities, no invalid chronic diseases, with some risk factors for ICD, like: short therapy with cephalosporin, abdominal surgery, age over 65 years, discharge from hospital seven days before ICD onset, initially considered medium severity, was treated orally with vancomycin.

Clinical aggravation imposed the association of metronidazole due to the occurrence of feverish, the persistence of 4-6 liquid stools/day, the occurrence of ascites and colon-ultrasound dilatation, the persistence of positive tests of Clostridium toxins in the stool despite the decrease in leukocytosis.

The cancellation of vancomycin and metronidazole was required due to the occurrence of the TM criteria: 8 liquid stools/day, colonoscopy with typical ICD images, TM radiologic criterion Cat scan - dilatation of ascending colon over $6 \mathrm{~cm}$, clinical criteria: fever 38.6 degrees Celsius, pulse $122 /$ minute, the occurrence of disease with leukocytes 31350 leukocytes $/ \mu 1$, BP $90 / 60 \mathrm{mmHg}$ (despite the support perfusion treatment), important abdominal distension, stasis, intestinal subocclusion aspect.

It was performed colonoscopy, despite the high risk of bowel perforation with suspected pathology associated colon that could explain clinical worsening and lack of response to vancomycin and metronidazole but it only revealed changes suggestive for ICD. Colonoscopy also revealed no perforations which justifies the continuation of drug therapy and surgical surveillance only.

The development of the TM criteria during treatment with vancomycin and metronidazole and the immediate clinical resolution under tigecycline suggests the presence of a CD strain resistant to vancomycin and metronidazole (not documented in terms of bacteria due to the inability of the laboratory to perform the culture in anaerobiosis and CMI for antibiotics) and sensible to tigecycline.

There are few data related to metronidazole and vancomycin resistance of CD. Freeman reported, in Europe, in 2015, 0.11\% Clostridium difficile strains resistant to metronidazole (MIC $\geq 8 \mathrm{mg} / \mathrm{L}$ ) and 2,2\% Clostridium difficile strains resistant to vancomycin $(\mathrm{MIC} \leq 2 \mathrm{mg} / \mathrm{L})$ [31].

We also take into consideration the gastric stasis which decreases effective oral medications (vancomycin, metronidazole) and increased efficiency, in such cases, intravenous medication (tigecycline).

It is noted that the initial testing was positive only toxin $\mathrm{B}$ and testing of clinical worsening were positive by both toxin A and B. This may be due to limited sensitivity Immunochromatographic tests or superinfection with a new strain of $C D$, with both toxins positive that may be responsible for worsening case.

The normalization of the number of leukocytes was not related to the clinical cure and did not prove a useful parameter for monitoring the evolution of the disease. The following criteria were much more useful: the number of stools and their consistency, temperature, serum albumin, imaging criteria - cat scans and colonoscopies.

The decrease in the number of leukocytes during treatment with vancomycin and metronidazole can be also due to the side effects of these antibiotics and their side effects anticipating the potential side effects to vancomycin - neutropenia and agrunolocytosis - rare - between 1/10.000 and 1/1.000 cases and to metronidazole- leucopenia and granulocitopenia - less frequent - between $1 / 1.000$ and $1 / 100$ cases 
and agranuloytosis very rare - under $1 / 10.000$ cases. When cancelling the treatment with vancomycin and metronidazole we discovered an increase in the number of leukocytes and neutrophils.

In conclusion, we emphasize effective intravenous medication in cases of the toxic megacolon from ICD and we consider necessary the performance of culture in anaerobiosis for CD as well as antibiogram with minimum inhibitory concentrations for vancomycin and metronidazole in severe cases or with clinical aggravation during treatment and reevaluating the indication of tigecycline from rescue therapy in associated therapy, first attempt, in ICD cases with toxic megacolon criteria.

\section{The particularity of case presented}

This case is an infection with Clostridium difficile in a immunocompetent men who presented clinical worsening of toxic megacolon with oral therapy with vancomycin and metronidazole, which performed favorably with injection therapy with tigecycline that raises the discussion related to the antibiotics sensitivity of the strain or superinfection with a high aggressiveness strain.

\section{Acknowledgement}

Thanks to Medicine and Pharmacy Research Center, "Dunărea de Jos" University of Galati, Faculty of Medicine for scientific support.

\section{References}

1. Cohen, S., Gerding, D., Johnson, S, Kelly, C., Loo, V., McDonald, C., Pepin, J. \& Wilcox, M. (2010). Clinical Practice Guidelines for Clostridium difficile Infection in Adults: 2010 Update by the Society for Healthcare Epidemiology of America (SHEA) and the Infectious Diseases Society of America (IDSA), Infection Control \& Hospital Epidemiology, Vol. 31, Issue 5, 431-455, DOI: http://dx.doi.org/10.1086/651706.

2. McDonald, L.C., Owings, M. \& Jernigan, D.B. (2006). Clostridium difficile infection in patients discharged from US short-stay hospitals, 19962003, Emerging Infectious Diseases Journal, Vol. 12, 409-415.

3. Redelings, M.D., Sorvillo, F. \& Mascola, L. (2007). Increase in Clostridium difficile-related mortality rates, United States, 1999-2004, Emerging Infectious Diseases Journal, Vol. 13, 1417-1419.

4. Burckhardt, F., Friedrich, A., Beier, D. \& Eckmanns, T. (2008). Clostridium difficile surveillance trends, Saxony, Germany, Emerging Infectious Diseases Journal, Vol. 14, pp. 691692.

5. Gravel, D., Miller, M., Simor, A., Taylor, G., Gardam, M., McGeer, A., Hutchinson, J., Moore, D., Kelly, S., Boyd, D. \& Mulvey, M. (2009). Health care-associated Clostridium difficile infection in adults admitted to acute care hospitals in Canada: a Canadian Nosocomial Infection Surveillance Program Study. Clin Infect Dis. 48(5), 568-576. doi: 10.1086/596703

6. Loo, V.G., Poirier, L., Miller, M.A., Oughton, M., Libman, M.D., Michaud, S., Bourgault, A.M., Nguyen, T., Frenette, C., Kelly, M., Vibien, A., Brassard, P., Fenn, S., Dewar, K., Hudson, T.J., Horn, R., Rene, P., Monczak, Y. \& Dascal, A. (2005). A predominantly clonal multiinstitutional outbreak of Clostridium difficileassociated diarrhea with high morbidity and mortality. $N$ Engl J Med. 353(23), 2442-2449. doi: 10.1056/NEJMoa051639 
7. Warny, M., Pepin, J., Fang, A., Killgore, G., Thompson, A., Brazier, J., Frost, E. \& McDonald, L.C. (2005). Toxin production by an emerging strain of Clostridium difficile associated with outbreaks of severe disease in North America and Europe. Lancet. 366(9491), 1079-1084. doi: 10.1016/S0140-6736(05)67420-X

8. Obuch-Woszczatynski, P., Dubiel, G., Harmanus, C., Kuijper, E., Duda, U., Wultanska, D., van Belkum, A. \& Pituch, H. (2013). Emergence of Clostridium difficile infection in tuberculosis patients due to a highly rifampicin-resistant PCR ribotype 046 clone in Poland. Eur J Clin Microbiol Infect Dis. 32(8), 1027-1030. doi: 10.1007/s10096-013-1845-5.

9. Rafila, A., Indra, A., Popescu, G.A., Wewalka, G., Allerberger, F., Benea, S., Badicut, I., Aschbacher, R. \& Huhulescu, S. (2014). Occurrence of Clostridium difficile infections due to PCR ribotype 027 in Bucharest, Romania. J Infect Dev Ctries. 8(6), 694-698. doi: 10.3855/ jidc.4435.

10. Benea, S., Popescu, G.A., Badicut, I., Florea, D., Petrache, D., Gavriliu, L. \& Rafila, A.. (2012). Clostridium difficile infections hospitalized in Romanian Institute of Infectious Diseases during the first three months of 2012, Poster Presentation, 4th International Clostridium difficile Symposium, September 20th to 22th, Bled, Slovenia.

11. Bartlett, J.G. \& Perl, T.M. (2005). The new Clostridium difficile--what does it mean? $N$ Engl J Med. 353(23), 2503-2505. doi: 10.1056/ NEJMe058221.

12. Arumilli, B.R., Koneru, P. \& Fayyaz, I. (2010). Toxic megacolon from hypervirulent Clostridium difficile infection (ribotype 027) following elective total knee replacement: an emerging challenge in modern health care. BMJ Case Rep. 2010. doi: 10.1136/bcr.06.2009.2017.

13. Autenrieth, D.M. \& Baumgart, D.C. (2012). Toxic megacolon. Inflamm Bowel Dis. 18(3), 584-591. doi: 10.1002/ibd.21847.

14. Dumitru, I.M., Dumitru, E., Rugina, S. \& Tuta, L.A. (2017). Toxic Megacolon - A Three Case Presentation, The Journal of Critical Care Medicine 3(1):39-44, DOI: 10.1515/jccm-2017-
0008.

15. Baroiu, L., Vasile, M.C., Baroiu, N. \& Drăgănescu, M. (2015), Aspects of Clostridium difficile infection in Second Department of the Infectious Diseases Hospital Galati, Proceedings of -11th Edition of the Scientific Days of the National Institute of Infectious Diseases "Prof. Dr. Matei Balş", Bucharest, October 14-16, ISSN 2457-8525, 65-66.

16. Marshak, R.H. \& Lester, L.J. (1950). Megacolon a complication of ulcerative colitis. Gastroenterology, 16:768-772.

17. Jalan, K.N., Sircus, W., Card, W.I., Bruce, J., Falconer, C.W., Small, W.P., Smith, A.N. \& McManus, J.P.A. (1967). Toxic dilatation of the colon - An experience of 55 cases. Gut. 8:633-638.

18. Wodzinski, M.A., Snowden, J.A. \& Reilly, J.T. (1994). Toxic megacolon complicating chemotherapy for acute myeloid leukemia. Postgrad Med J., 70(830):921-3.

19. Hayes-Lattin, B.M., Curtin, P.T., Fleming, W.H., Leis, J.F., Stepan, D.E., Schubach, S. \& Maziarz, R.T. (2002). Toxic megacolon: a life-threatening complication of high-dose therapy and autologous stem cell transplantation among patients with AL amyloidosis. Bone Marrow Transplant. 30(5), 279-285. doi: 10.1038/sj.bmt.1703627.

20. Fitzgerald, S.C., Conlon, S., Leen, E. \& Walsh, T.N. (2009). Collagenous colitis as a possible cause of toxic megacolon. Ir J Med Sci. 178(1), 115-7.

21. Bains, S., Lloyd, G.M., Sutton, C.D., West, K. \& Miller, A.S. (2009). A case of toxic megacolon in a patient with collagenous colitis. Tech Coloproctol. 13(2), 165-6.

22. Umehara, Y., Kudo, M. \& Kawasaki, M. (2010). Endoscopic findings of intestinal Behcet's disease complicated with toxic megacolon. Endoscopy. 42 Suppl 2, E173-174. doi: 10.1055/ s-0028-1103446.

23. Adorian, C., Khoury, G., Tawil, A. \& Sharara, A. (2003). Behcet's disease complicated by toxic megacolon. Dig Dis Sci. 48(12), 2366-2368.

24. Sheth, S.G. \& LaMont, J.T. (1998). Toxic megacolon. Lancet. 351(9101), 509-513. doi: 10.1016/S0140-6736(97)10475-5

25. Sayedy, L., Kothari, D. \& Richards, R.J. (2010). 
Toxic megacolon associated Clostridium difficile colitis. World J Gastrointest Endosc. 2(8), 293297. doi: 10.4253/wjge.v2.i8.293.

26. Sidebotham, E.L., Sepkowitz, K., Price, A.P., Steinherz, P.G., La Quaglia, M.P. \& Kayton, M.L. (2010). Eradication of cryptosporidium from a defunctionalized colon limb by refeeding stoma effluent. J Pediatr Surg. 45(1), E33-36. doi: 10.1016/j.jpedsurg.2009.10.091.

27. Shimada, Y., Iiai, T., Okamoto, H., Suda, T., Hatakeyama, K., Honma, T. \& Ajioka, Y. (2003). Toxic megacolon associated with cytomegalovirus infection in ulcerative colitis. $J$ Gastroenterol. 38(11), 1107-1108. doi: 10.1007/ s00535-003-1217-5.

28. Hung, C.W., Wu, W.F. \& Wu, C.L. (2009). Rotavirus gastroenteritis complicated with toxic megacolon. Acta Paediatr. 98(11), 1850-1852. doi: 10.1111/j.1651-2227.2009.01444.x.

29. Mohite, U., Kell, J., Haj, M.A., O’Brien, C., Kundu, S., Rees, J. \& Burnett, A.K. (2007). Invasive aspergillosis localised to the colon presenting as toxic megacolon. Eur $J$ Haematol. 78(3), 270-273. doi: 10.1111/j.16000609.2006.00812.x

30. Lin, B. \& Y Wu, G. (2016). Toxic Megacolon. from http://emedicine.medscape.com/ article/181054-overview.

31. Freeman, J., Vernon, J., Morris, K., Nicholson, S., Todhunter, S., Longshaw, C. \& Wilcox, M.H. (2015). Pan-European longitudinal surveillance of antibiotic resistance among prevalent Clostridium difficile ribotypes. Clin. Microbiol. Infect. 21, doi:10.1016/j.cmi.2014.09.017. 